\title{
BMJ
}

\section{Lateral wedge insoles for medial knee osteoarthritis: 12 month randomised controlled trial}

\author{
Kim L Bennell, professor, ${ }^{1}$ Kelly-Ann Bowles, research scientist, ${ }^{1}$ Craig Payne, senior lecturer, ${ }^{2}$ Flavia Cicuttini, \\ professor, ${ }^{3}$ Elizabeth Williamson, postdoctoral fellow, ${ }^{3,4}$ Andrew Forbes, professor, ${ }^{3}$ Fahad Hanna, \\ postdoctoral fellow, ${ }^{3,5}$ Miranda Davies-Tuck, postdoctoral fellow, ${ }^{3}$ Anthony Harris, professor, ${ }^{6}$ Rana S \\ Hinman, associate professor ${ }^{1}$
}

\begin{abstract}
${ }^{1}$ Centre for Health, Exercise and Sports Medicine, Department of Physiotherapy, University of Melbourne, Parkville, 3010 VIC, Australia

${ }^{2}$ Department of Podiatry, School of Human Biosciences, LaTrobe University, Melbourne, Victoria, Australia
\end{abstract}

${ }^{3}$ Department of Epidemiology and Preventive Medicine, Alfred Hospital, Monash University, Melbourne, Victoria, Australia

${ }^{4}$ MEGA Epidemiology, School of Population Health, University of Melbourne, Victoria, Australia

${ }^{5}$ Baker IDI Heart and Diabetes Institute, Melbourne, Victoria, Australia

${ }^{6}$ Health Economics Unit, Monash University, Melbourne

Correspondence to: K L Bennell k.bennell@unimelb.edu.au

Cite this as: BMJ 2011;342:d2912 doi:10.1136/bmj.d2912

\section{ABSTRACT}

Objective To assess the effect of lateral wedge insoles compared with flat control insoles on improving symptoms and slowing structural disease progression in medial knee osteoarthritis.

Design Randomised controlled trial.

Setting Community in Melbourne, Australia.

Participants 200 people aged 50 or more with clinical and radiographic diagnosis of mild to moderately severe medial knee osteoarthritis.

Interventions Full length 5 degree lateral wedged insoles or flat control insoles worn inside the shoes daily for 12 months.

Main outcome measures Primary symptomatic outcome was change in overall knee pain (past week) measured on an 11 point numerical rating scale. Primary structural outcome was change in volume of medial tibial cartilage from magnetic resonance imaging scans. Secondary clinical outcomes included changes in measures of pain, function, stiffness, and health related quality of life. Secondary structural outcomes included progression of medial cartilage defects and bone marrow lesions.

Results Between group differences did not differ significantly for the primary outcomes of change in overal pain $(-0.3$ points, $95 \%$ confidence intervals -1.0 to 0.3$)$ and change in medial tibial cartilage volume $\left(-0.4 \mathrm{~mm}^{3}\right.$, $95 \%$ confidence interval -15.4 to 14.6 ), and confidence intervals did not include minimal clinically important differences. None of the changes in secondary outcomes showed differences between groups.

Conclusion Lateral wedge insoles worn for 12 months provided no symptomatic or structural benefits compared with flat control insoles.

Trial registration Australian New Zealand Clinical Trials Registry ACTR12605000503628 and ClinicalTrials.gov NCT00415259.

\section{INTRODUCTION}

Knee osteoarthritis, which most commonly affects the medial compartment, is a chronic joint disorder that imposes a major healthcare burden. ${ }^{1}$ As no cure exists, traditional management aims to reduce pain, improve function, and enhance quality of life while minimising the adverse effects of therapy. Non-drug conservative interventions are considered the first line approach to osteoarthritis management. ${ }^{2}$ However, given that a substantial proportion of patients with knee osteoarthritis experience progression of structural disease, ${ }^{3}$ contemporary management also aims to reduce structural deterioration. Research since early 2000 shows that increased medial knee joint loading is an important risk factor for disease progression of medial osteoarthritis. ${ }^{4}$ Thus non-surgical treatments that reduce medial load on the knee warrant investigation.

Lateral wedge shoe insoles are an inexpensive readily available treatment that has been shown to reduce medial knee load. ${ }^{56}$ Wedged insoles are recommended by 13 of 14 international guidelines for knee osteoarthritis ${ }^{2}$; however, the limited research available has failed to show any significant impact of lateral wedge insoles on the symptoms of osteoarthritis. The few randomised controlled trials that have been done are limited by factors such as small sample size, short intervention period, use of suboptimal lateral wedge design, or a heterogeneous cohort with osteoarthritis. ${ }^{7-10}$ Importantly, only one trial has evaluated the effects of lateral wedge insoles on joint structure. ${ }^{9}$ The non-significant finding in this study may be related to the use of radiology to measure structural change, which is less sensitive than magnetic resonance imaging, ${ }^{11} 12$ or to the use of heel wedges, which do not reduce medial load as much as full length wedges. ${ }^{13}$

We carried out a randomised controlled trial to assess the efficacy of lateral wedge insoles compared with control insoles worn daily for 12 months on improving symptoms and slowing structural disease progression in people with medial knee osteoarthritis.

\section{METHODS}

We recruited participants from the community through advertisements in local clubs and the print and radio media in metropolitan Melbourne, Australia, between May 2005 and July 2008. Inclusion criteria were age 50 years or more, average knee pain on walking more than 3 on an 11 point scale $(0=$ no pain; $10=$ worst pain possible) at telephone screening, pain 
located over the medial knee compartment, evidence of osteophytes in the medial compartment or medial joint space narrowing on an $\mathrm{x}$ ray film, ${ }^{14}$ and radiological knee alignment of 185 degrees or less (corresponding to a mechanical axis angle of $\leq 182$ degrees and indicating neutral to varus (bow leg) knee alignment on an $\mathrm{x}$ ray film of the whole leg). ${ }^{15}$ All participants provided written informed consent.

Exclusion criteria were questionable or advanced radiographic knee osteoarthritis (Kellgren and Lawrence grades 1 and 4$),{ }^{16}$ predominant patellofemoral joint symptoms on clinical examination (location of pain, pain provoking activities, tenderness on palpation, and pain during mobilisation of the patellar), ${ }^{17}$ knee surgery or intra-articular corticosteroid injection within six months, current or past (within four weeks) use of oral corticosteroids, systemic arthritic conditions, history of knee arthroplasty or osteotomy, other musculoskeletal or neurological condition affecting leg function, disease of the ankle or foot precluding the use of insoles, use of foot orthotics within the past six months, usual footwear unable to accommodate insoles, contraindications to magnetic resonance imaging, planning to start other treatment for knee osteoarthritis, and regular use of a gait aid.

\section{Procedures}

We carried out a double blind randomised controlled trial over 12 months, the methodology of which has been described previously. ${ }^{18}$ Potential participants underwent telephone screening followed by standardised semiflexed standing posteroanterior knee radiology, to assess the severity of knee osteoarthritis and knee joint alignment. A physiotherapist or medical practitioner and a podiatrist then carried out a screening clinical examination. Participants were stratified by disease severity (Kellgren and Lawrence grades 2 and $3^{16}$ ) and sex and randomly allocated in permuted blocks of 6 to 12 to either the lateral wedge insole or the control insole group. An independent investigator used a computer program to generate the randomisation sequence a priori. Allocation was sealed in opaque and consecutively numbered envelopes held centrally. Envelopes were opened sequentially by an independent person. Participants were informed that two types of insoles were being compared but the insoles and study hypotheses were not described.

\section{Interventions}

Participants wore the insoles bilaterally in their own shoes every day. They were provided with two pairs of insoles, which were replaced every four months. The lateral wedge ( 5 degrees) insoles were made of high density ethyl vinyl acetate (similar to the midsole in a running shoe) and were wedged along the lateral border of the foot (Foot Function, New Zealand, see web extra). We chose a 5 degree wedge because greater wedging is less likely to be tolerated by the wearer ${ }^{6}$ and is difficult to accommodate within a normal shoe. The control insoles were made of easily compressible low density ethyl vinyl acetate but with no wedging (Foot Function, New Zealand).

\section{Outcome measures}

A blinded examiner assessed the participants at baseline and 12 months. In participants with bilateral knee osteoarthritis the most symptomatic eligible knee was assessed. Baseline demographic information was collected and participants rated their expectation of a beneficial effect with insole treatment on an ordinal scale from 1 to 5 ( $0=$ no effect at all, $5=$ complete recovery), with higher scores indicating higher expectations.

\section{Symptomatic measures}

The primary symptomatic measure was overall average knee pain (past week) using an 11 point numerical rating scale $\left(0=\right.$ no pain, $10=$ worst pain possible) ${ }^{19}$ This has well accepted clinimetric properties and is widely used and recommended for clinical trials on knee osteoarthritis. ${ }^{19-22}$

Secondary symptomatic measures included pain on walking (measured on the 11 point scale), pain, stiffness, and physical function subscales of the Western Ontario and McMaster Universities osteoarthritis index, ${ }^{23}$ assessment of quality of life instrument, ${ }^{24}$ and patient perceived global change in pain and in physical function (compared with baseline) measured on a 5 point ordinal scale and dichotomised into improvement (slightly better and much better) and no improvement (much worse, slightly worse, and no change). We also measured levels of physical activity using two methods: the physical activity scale for the elderly questionnaire, with higher scores indicating greater physical activity, ${ }^{25}$ and the average number of steps taken per day, as measured by a pedometer (KH-005; Omron Healthcare, Japan), worn for one week on two occasions. Participants recorded use of and discomfort with insoles daily in a log book (returned on a monthly basis) and using an 11 point numerical rating scale at follow-up. Adverse events and cointerventions were recorded in the log book and by open probe questioning at follow-up.

\section{Structural measures}

The primary structural outcome measure was the volume of cartilage in the medial tibial compartment on magnetic resonance imaging. Images of the knee in the sagittal plane were obtained on a T1 weighted whole body unit as previously described. ${ }^{26} \mathrm{We}$ defined the volume of the medial tibial cartilage plate by manually drawing disarticulation contours around the cartilage boundary on each section. Data were resampled by bilinear and cubic interpolation (area of 312 and $312 \mu \mathrm{m}$ and $1.5 \mathrm{~mm}$ thickness, continuous sections) for final three dimensional rendering. We determined the volume of medial tibial cartilage plate by summing the pertinent voxels within the resultant binary volume. Two trained blinded observers independently determined the measurements. The coefficient of variation for the cartilage volume measure was $3.4 \% .{ }^{27} \mathrm{To}$ control medial tibial cartilage volume for bone size, we 
Assessed for eligibility by telephone $(n=1923)$

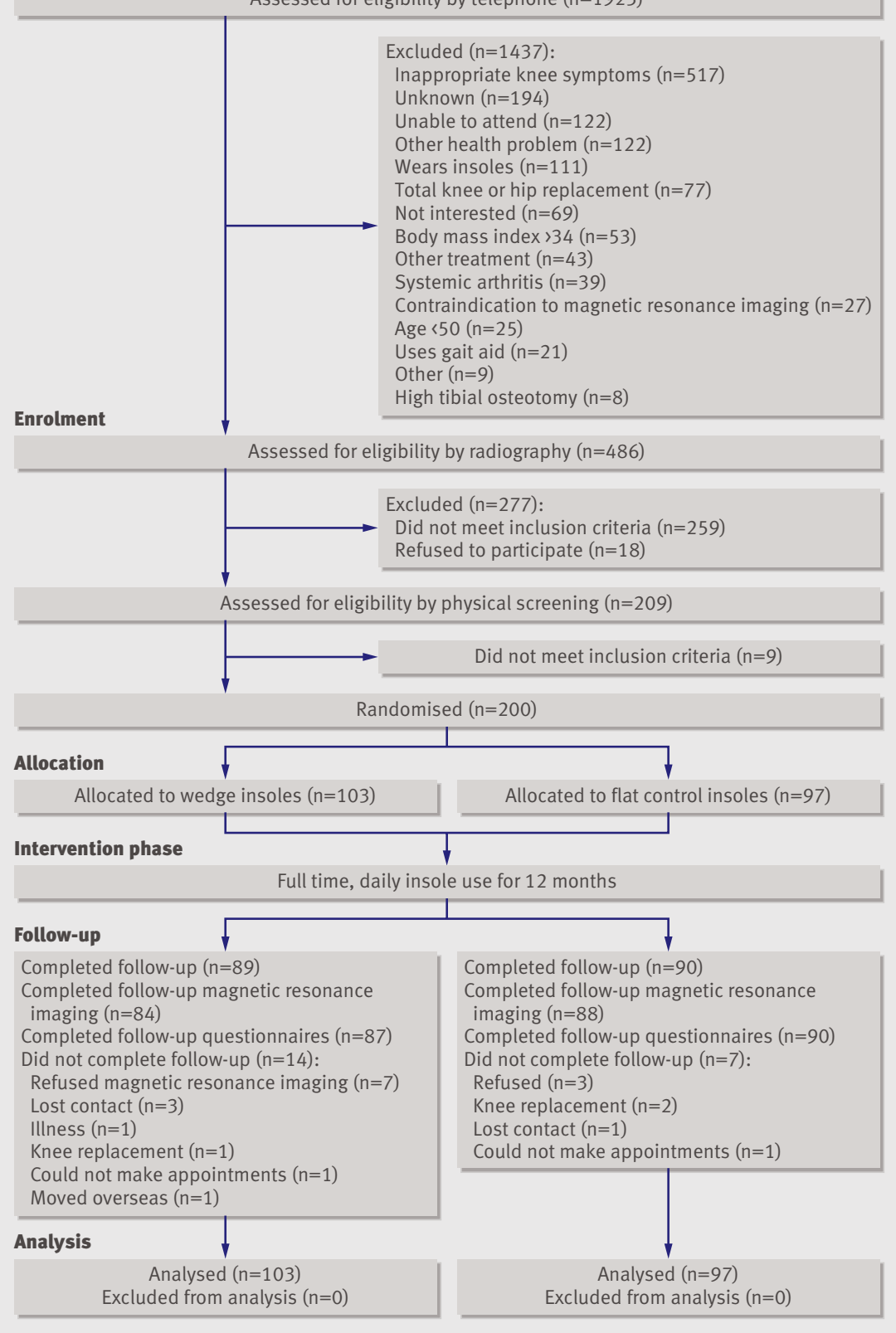

Fig 1| Flow of participants through trial

determined the cross sectional area of the medial tibial plateau by creating an isotropic volume from the input image, which was reformatted in the axial plane, using the software program Osiris. The area was directly measured from this axial image as described previously. ${ }^{28}$ By subtracting the follow-up volume from baseline volume and dividing by time between scans we determined the annual change in tibial cartilage volume. This was also expressed as a percentage of initial volume.

Secondary structural outcomes included cartilage defects, graded with a classification system on a 0 to 4 scale, as described previously ${ }^{2930}$ for medial tibial and femoral compartments: grade 0, normal cartilage; grade 1 , focal blistering and intracartilaginous low signal intensity area with an intact surface and bottom; grade 2 , irregularities on the surface or bottom and loss of thickness of less than 50\%; grade 3, deep ulceration with loss of thickness of more than $50 \%$; and grade 4 , full thickness cartilage wear with exposure of subchondral bone. The measurement was carried out by one trained observer, who measured all images in duplicate on separate occasions. Progression of medial tibiofemoral cartilage defects was determined if the cartilage defect score increased by at least 1 from baseline to follow-up in the medial tibial or medial femoral compartment.

Bone marrow lesions were defined as areas of increased signal intensity adjacent to subcortical bone present in either the medial distal femur or the proximal tibia, and their size was graded on a 0 to 2 scale as described previously from coronal T2 fat saturated images $^{31}$ : grade 0 , absence of a lesion; grade 1 , lesion encompassed up to $25 \%$ of the width of the tibial or femoral cartilage being examined from coronal images; and grade 2, lesion encompassed more than $25 \%$ of the width of the tibial or femoral cartilage being examined from coronal images. We also recorded the number of slices the bone marrow lesions encompassed. To provide the medial tibiofemoral bone marrow lesion we multiplied the bone marrow lesion grade (0-2) by the number of slices for the medial femoral and medial tibial compartment separately, which was then summed. Progression of bone marrow lesions was defined if there was an increase in bone marrow lesion score of 1 or more over the periodthat is, follow-up tibiofemoral bone marrow lesion score minus the baseline lesion score of 1 or more. This scoring system is a valid measure of bone marrow lesions as it has been shown to be sensitive to change and to detect clinically important outcomes. ${ }^{32} 33$

\section{Magnetic resonance imaging machines}

We used two different magnetic resonance imaging machines; initially a Philips machine (Eindhoven, Netherlands) followed by a GE machine (Signa Advantage HiSpeed GE Medical Systems, Milwaukee, WI), owing to decommissioning of the Philips machine. In total, $117(68 \%)$ participants were scanned on the same machine at baseline and follow-up (102 had both scans on the Philips machine and 15 had both scans on the GE machine), whereas 54 (32\%) participants were scanned on the Philips machine at baseline and the GE machine at follow-up.

A validity study confirmed no systematic difference with change of machines. Fifteen participants underwent scans of one knee using both machines. The mean medial tibial cartilage volume as measured on the Philips and GE machines was 1706.3 (SD 361.2) $\mathrm{mm}^{3}$ and 1719.3 (SD 394.4) $\mathrm{mm}^{3}$, respectively $(\mathrm{P}>0.05)$. The intraclass correlation coefficient from a one way analysis of variance was 0.98 (95\% confidence interval 0.95 to 0.99 ), showing excellent absolute agreement between measures. A Bland-Altman plot of the difference (Philips-GE machine) versus average measurements showed the mean to be $-13 \mathrm{~mm}^{3}$, with 
Table 1|Baseline characteristics of participants, by treatment group. Values are numbers (percentages) unless stated otherwise

\begin{tabular}{|c|c|c|}
\hline Variable & Wedge insoles $(n=103)$ & Control insoles $(n=97)$ \\
\hline Mean (SD) age (years) & $63.3(8.1)$ & $65.0(7.9)$ \\
\hline Women & $62(60)$ & $56(58)$ \\
\hline Mean (SD) body mass index & $28.1(4.2)$ & $30.4(5.6)$ \\
\hline Mean (SD) symptom duration (years) & $7.1(7.9)$ & $7.5(7.7)$ \\
\hline \multicolumn{3}{|l|}{ Duration of symptoms (years): } \\
\hline$<1$ & $11(11)$ & $5(5)$ \\
\hline 1 to $<5$ & $42(41)$ & $40(41)$ \\
\hline 5 to $<10$ & $21(21)$ & $23(24)$ \\
\hline$\geq 10$ & $28(28)$ & $29(30)$ \\
\hline Unilateral symptoms & $33(32)$ & $37(38)$ \\
\hline \multicolumn{3}{|l|}{ Radiographic disease severity: } \\
\hline Grade 2 & $49(48)$ & $46(47)$ \\
\hline Grade 3 & $54(52)$ & $51(53)$ \\
\hline \multicolumn{3}{|l|}{ Location of osteophytes: } \\
\hline Medial compartment & $103(100)$ & $93(96)$ \\
\hline Lateral compartment & $23(22)$ & $21(22)$ \\
\hline \multicolumn{3}{|l|}{ Location of joint space narrowing: } \\
\hline Medial compartment & $99(96)$ & $96(99)$ \\
\hline Lateral compartment & $80(78)$ & $77(79)$ \\
\hline Mean (SD) anatomical alignment (degrees) & $181(3)$ & $181(3)$ \\
\hline \multicolumn{3}{|l|}{ Current drug use: } \\
\hline Analgesia & $9(9)$ & $13(13)$ \\
\hline Non-steroidal anti-inflammatory drugs & $15(15)$ & $26(27)$ \\
\hline Cyclo-oxygenase- 2 inhibitors & $2(2)$ & $4(4)$ \\
\hline Glucosamine products & $34(33)$ & $30(31)$ \\
\hline \multicolumn{3}{|l|}{ Past treatments: } \\
\hline Arthroscopy & $45(44)$ & $43(44)$ \\
\hline Physiotherapy & $16(16)$ & $11(11)$ \\
\hline Exercise & $3(3)$ & $2(2)$ \\
\hline Cortisone injection & $3(3)$ & $3(3)$ \\
\hline
\end{tabular}

Not all numbers add up to totals owing to missing data.

$95 \%$ limits of agreement of -152 to $126 \mathrm{~mm}^{3}$. The standard error of measurement was calculated as $49.3 \mathrm{~mm}^{3}$. To compare between machine repeatability with within machine repeatability, 12 of these 15 participants underwent a second measurement on the Philips machine, yielding a standard error of measurement of $33.3 \mathrm{~mm}^{3}$, with $95 \%$ limits of agreement of -75.6 to $106.5 \mathrm{~mm}^{3}$. These limits indicate comparable but slightly less reproducibility between machines than within machines.

\section{Sample size}

Overall, 126 participants were required to detect a minimal clinically important difference of 1.5 for change in pain between groups, ${ }^{34}$ assuming a standard deviation of 3 (based on previous data ${ }^{3536}$ ), with $80 \%$ power at a $5 \%$ significance level. We have shown that the mean rate of tibial cartilage loss is 5.3\% (SD 5.2\%) per year in knee osteoarthritis. ${ }^{27}$ Data suggest that a clinically beneficial outcome with lateral wedge insoles would be to reduce the rate of cartilage loss to less than $3 \%$ per annum, as this is associated with a reduced risk for arthroplasty within four years. ${ }^{37}$ Thus we required a minimum of 160 participants to detect a difference of
$2.3 \%$ between groups, with $80 \%$ power at a $5 \%$ significance level. We increased the sample size to 200 to account for dropouts and to allow for at least $80 \%$ power for both the primary outcomes.

\section{Statistical analysis}

A blinded statistician carried out the analysis, which was by intention to treat. All analyses were done using Stata (Version 11), and we considered P values of less than 0.05 to be significant. For continuous outcome measures we used linear regression modelling adjusted for baseline values of the outcome to compare differences in mean change (baseline minus follow-up) between groups. Results are presented as estimated differences with $95 \%$ confidence intervals. The analysis of change in medial cartilage volume was repeated with further adjustment for age, sex, body mass index, medial tibial bone size, and magnetic resonance imaging machine. We summarised the total medial bone marrow lesion scores as the median change from baseline (interquartile range) and compared scores between groups using the difference in median change. Using log binomial regression we compared global change in pain and in physical function and progression of cartilage defects between groups. Results are presented as relative risks with $95 \%$ confidence intervals. We used bootstrap confidence intervals based on 5000 replications to compare changes in the size of bone marrow lesions.

To account for missing data (31/400 pain measures and 29/400 medial tibial cartilage volume measures, $7 \%$ of total baseline and follow-up dataset for each measure) we carried out a sensitivity analysis using single mean imputation of missing baseline measures ${ }^{38}$ and multiple imputation of missing follow-up measures, assuming data are missing at random and follow a multivariate ${ }^{39}$ normal distribution. As results were unchanged we present complete case analyses.

We undertook a second sensitivity analysis to estimate the between group difference that would occur if all participants adhered completely to their allocated treatment. For these analyses adherence was measured by the number of days the insoles were worn as reported in the log books. A $t$ test was used to compare adherence between the groups. For each of the two primary outcomes we used a two stage least squares instrumental variables approach. ${ }^{40}$ This involved a regression model of the outcome measure adjusted for the baseline value and adherence, and a second regression model of adherence was adjusted for randomised group. The two regression models were fitted simultaneously and we estimated the effect of the lateral wedged insoles under full adherence.

\section{RESULTS}

Of the 1923 volunteers, 1437 (74.7\%) were ineligible or did not wish to participate (fig 1). In total 200 participants (103 lateral wedge insoles, 97 control insoles) were randomised and 179 (89 lateral wedge insoles, 90 control insoles; $90 \%$ ) completed the trial. Baseline descriptive characteristics of participants indicated no 


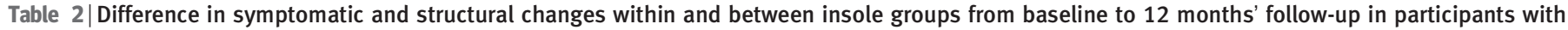
medial knee osteoarthritis

\begin{tabular}{|c|c|c|c|c|c|c|c|}
\hline \multirow[b]{2}{*}{ Outcome } & \multicolumn{2}{|c|}{ Mean (SD) week 0} & \multicolumn{2}{|c|}{ Mean (SD) week 52} & \multicolumn{2}{|c|}{$\begin{array}{l}\text { Mean (SD) difference within } \\
\text { groups (week 0-week 52) }\end{array}$} & \multirow{2}{*}{$\begin{array}{c}\begin{array}{c}\text { Mean }(95 \% \mathrm{Cl}) \\
\text { difference } \\
\text { between groups } \\
\text { (week 0-week } 52)^{\star}\end{array} \\
\begin{array}{c}\text { Wedge insoles } \\
\text {-control insoles }\end{array}\end{array}$} \\
\hline & $\begin{array}{l}\text { Wedge insoles } \\
\quad(n=103)\end{array}$ & $\begin{array}{c}\text { Control insoles } \\
(n=97)\end{array}$ & $\begin{array}{l}\text { Wedge insoles } \\
\quad(n=89)\end{array}$ & $\begin{array}{l}\text { Control insoles } \\
(n=90)\end{array}$ & $\begin{array}{l}\text { Wedge } \\
\text { insoles }\end{array}$ & $\begin{array}{l}\text { Control } \\
\text { insoles }\end{array}$ & \\
\hline \multicolumn{8}{|l|}{ Secondary measures } \\
\hline \multicolumn{8}{|l|}{ Primary outcomes } \\
\hline Average pain† (0-10) & $4.0(2.1)$ & $4.3(1.9)$ & $3.1(2.1)$ & $3.1(2.3)$ & $0.9(2.1)$ & $1.3(2.4)$ & $-0.3(-1.0$ to 0.3$)$ \\
\hline $\begin{array}{l}\text { Medial tibial cartilage } \\
\text { volume }\left(\mathrm{mm}^{3}\right)\end{array}$ & $1550(452)$ & $1520(439)$ & $1513(480)$ & $1483(432)$ & $43(45)$ & $43(53)$ & $-0.4(-15.4$ to 14.6$)$ \\
\hline Pain on walkingt $(0-10)$ & $4.2(2.2)$ & $4.3(1.9)$ & $3.2(2.1)$ & $3.0(2.5)$ & $0.9(2.2)$ & $1.4(2.6)$ & $-0.3(-1.0$ to 0.4$)$ \\
\hline \multicolumn{8}{|l|}{ WOMAC‡: } \\
\hline Pain $(0-20)$ & $7.1(3.0)$ & $7.2(2.9)$ & $6.4(3.3)$ & $6.2(3.2)$ & $0.7(2.7)$ & $1.2(3.1)$ & $-0.4(-1.2$ to 0.4$)$ \\
\hline Function (0-68) & $23.7(12.2)$ & $23.6(10.9)$ & $20.8(12.2)$ & $20.1(11.6)$ & $3.1(9.0)$ & $4.0(11.2)$ & $-0.7(-3.6$ to 2.2$)$ \\
\hline Stiffness $(0-8)$ & $4(2)$ & $4(1)$ & $3(2)$ & $3(2)$ & $0.4(1.4)$ & $0.6(1.7)$ & $-0.3(-0.7$ to 0.2$)$ \\
\hline $\begin{array}{l}\text { Health related quality of } \\
\text { life }(-0.04-1.00) \S\end{array}$ & $0.7(0.2)$ & $0.7(0.2)$ & $0.7(0.2)$ & $0.7(0.2)$ & $-0.02(0.11)$ & $-0.01(0.13)$ & $-0.01(-0.05$ to 0.02$)$ \\
\hline $\begin{array}{l}\text { Physical activity scale } \\
\text { for elderly }(0-400)\end{array}$ & $182(81)$ & $162(68)$ & $167(83)$ & $167(88)$ & $16(77)$ & $1(83)$ & $7.8(-15.4$ to 30.9$)$ \\
\hline No of daily steps & $7908(3712)$ & 7562 (3593) & 8059 (4946) & $6688(4106)$ & $-14(3189)$ & $673(3217)$ & -797.9 (-1966 to 370.2$)$ \\
\hline
\end{tabular}

WOMAC $=$ Western Ontario and MCMaster Universities osteoarthritis index.

Differences within and between groups concern only participants with baseline and follow-up data.

*Adjusted for baseline value of variable.

†Assessed using numerical rating scale, with higher scores indicating worse pain.

¥Higher scores indicating worse pain, function, and stiffness.

§Assessed using assessment of quality of life instrument, with higher scores indicating better quality of life.

differences between groups (table 1). Characteristics of the 21 participants lost to follow-up were similar to those completing the study (data not shown). The participants' expectation of treatment outcomes was similar between groups $(\mathrm{P}=0.51)$, with $54 \%(\mathrm{n}=97)$ of 180 participants who provided these data expecting moderate improvement, 25\% ( $\mathrm{n}=45)$ expecting large improvement, and $2 \%(n=4)$ expecting complete recovery.

\section{Outcome measures}

The between group difference for the primary outcome of change in overall average pain did not differ significantly $(-0.3$ points, $95 \%$ confidence interval, -1.0 to 0.3 ), table 2). Both groups showed small mean reductions in pain over time, but these reductions were smaller than the minimal clinically important difference. ${ }^{34}$ The between group difference for the primary outcome of change in medial tibial cartilage volume over 12 months did not differ significantly when expressed in absolute terms $\left(-0.4 \mathrm{~mm}^{3}, 95 \%\right.$ confidence interval -15.4 to 14.6 , table 2) or when expressed as the percentage change. Lateral wedge insoles were associated with a mean reduction of $3.0 \%$ (SD 3.1\%) in cartilage volume compared with $2.8 \%(3.5 \%)$ for control insoles (mean difference $0.2 \%, 95 \%$ confidence interval $-0.8 \%$ to $1.2 \%)$. Further adjustment for covariates (sex, age, body mass index, bone size, and magnetic resonance imaging machine) resulted in an estimate of between group difference in change in medial tibial cartilage volume of $-0.7 \mathrm{~mm}^{3}$ (95\% confidence interval -16.7 to 15.4 ).
The primary results did not change under a hypothetical scenario of complete adherence to treatment (allocated insoles worn daily for a year by every patient). In this scenario, the between group difference in mean change in average pain was estimated as -1.6 points (95\% confidence interval -5.6 to $2.5, \mathrm{P}=0.444)$, and for change in medial tibial cartilage volume was estimated as $-25.5 \mathrm{mg}^{3}$ (95\% confidence interval -130.6 to 80.0, $\mathrm{P}=0.634)$.

Lateral wedge insoles had similar effects to control insoles across all secondary outcome measures (tables 2 and 3). Although most participants reported improved pain and function after treatment there was no difference between groups for global change scores (table 3). Changes in secondary structural outcomes were also similar between groups (table 3). A relatively small number of participants showed progression of medial tibial or femoral cartilage defects over 12 months. Changes in the size of bone marrow lesions were also similar between groups, with the median change being 0 (interquartile range -2-3) for the lateral wedge insole group and 0 (-2-2) for the control insole group, with an estimated difference in median changes of 0 (95\% bootstrap confidence interval -0.31 to 0.31 ).

\section{Adherence, insole comfort, adverse events, and} cointerventions

Log book completion rates were $69 \%$ for the lateral wedge insole group and 73\% for the control insole group. The mean duration of daily insole use was significantly lower in the lateral wedge insole group than in the control insole group (6.8 (SD 4.3) hours per day $v$ 
Table $3 \mid$ Participants reporting global improvement in symptoms and showing progression of medial tibial and femoral cartilage defects according to insole group. Values are numbers (percentages) unless stated otherwise

\begin{tabular}{lccc} 
Outcome & $\begin{array}{c}\text { Wedge insoles } \\
(\mathrm{n}=\mathbf{8 2})\end{array}$ & $\begin{array}{c}\text { Control insoles } \\
(\mathrm{n}=85)\end{array}$ & $\begin{array}{c}\text { Relative risk } \\
(95 \mathrm{Cl})\end{array}$ \\
\hline Global improvement in pain* & $57(70)$ & $55(64)$ & $1.09(0.88$ to 1.35) \\
\hline Global improvement in function* & $46(56)$ & $53(62)$ & $0.91(0.71$ to 1.17$)$ \\
\hline Progression of medial tibial cartilage defects & $13(16)$ & $18(21)$ & $0.75(0.39$ to 1.43$)$ \\
\hline Progression of medial femoral cartilage defects & $16(20)$ & $18(21)$ & $0.92(0.51$ to 1.68$)$ \\
\hline
\end{tabular}

*Participants rating themselves as much better or slightly better.

9.1 (3.8) hours per day, $\mathrm{P}<0.01$ ) and was relatively consistent over the study (fig 2). Self reported adherence with insole use was also lower in the lateral wedge insole group, reflecting log book data (table 4).

More participants reported problems with the lateral wedge insoles $(42 / 89,47 \%)$ than with the control insoles $(21 / 90,23 \%$, table 4). Lateral wedge insoles were more likely to be associated with back and foot pain and to be difficult to fit into shoes than the control insoles. Lateral wedge insoles were also rated as less comfortable. Around half of the participants stated that the lateral wedge insole was associated with some degree of discomfort compared with $30 \%$ of participants in the control group. Severe or very severe discomfort was rated by $7(10 \%)$ in the lateral wedge insole group compared with $1(1 \%)$ in the control insole group.

Use of analgesic, non-steroidal anti-inflammatory drugs and glucosamine preparations was similar between groups (table 4). Cointerventions were reported by $17 / 89(19 \%)$ participants in the lateral wedge insole group and 14/90 (16\%) participants in the control insole group (table 4 ), with a similar number and type of cointerventions across groups.

\section{DISCUSSION}

Our randomised controlled trial showed that lateral wedge insoles compared with flat control insoles worn for 12 months provided no additional benefit in alleviating symptoms or slowing disease progression in older adults ( $\geq 50$ years) with mild to moderately severe medial knee osteoarthritis. Our results do not support the recommendations of clinical guidelines advocating the use of lateral wedge insoles for the management of medial knee osteoarthritis. ${ }^{2}$

\section{Explanation of results}

Most of our participants had varus knee alignment, a factor known to increase the risk of structural disease progression $^{41}$ and thus rendering our cohort an osteoarthritis subgroup ideally suited to disease modifying interventions. Our lateral wedge insoles had no significant effect on structural change over time. Similar annual loss of medial tibial cartilage volume was observed in both treatment groups (2.8-3.0\%) and this is within the range of annual cartilage loss in knee osteoarthritis reported by longitudinal studies. ${ }^{42}$ Our non-significant findings occurred even though we used lateral wedge insoles known to reduce medial knee load during walking. ${ }^{5}$ Specifically, we have previously reported a significant reduction of $5.4 \%$ (SD $5.2 \%$ ) in the knee adduction moment during walking (a measure of medial knee joint compartment load) with these particular lateral wedge insoles. ${ }^{5}$ Given the established relation between knee load and risk of structural deterioration over time, ${ }^{4}$ it is unclear why our lateral wedge insoles did not reduce the rate of cartilage loss. It is possible our 12 month intervention period was not long enough to detect a disease modifying effect.

It is also possible that our use of the participant's own non-standardised shoes was a factor. Different shoe types can influence the biomechanical and clinical effectiveness of insoles, and in particular shoes with heels can attenuate their effects. ${ }^{43}$ Participants were encouraged to wear the insoles in their usual shoes; however, we were unable to assess adherence to this recommendation. This was because of the difficulty in classifying the myriad shoe types in existence and the burden such daily recording would have imposed on participants, with its subsequent impact on adherence and retention in the study.

The amount and type of physical activity could potentially mediate the effect of the insoles, given that physical activity levels will influence cumulative load at the knee. However, given that physical activity levels as measured from either questionnaire or a pedometer did not differ between the groups during the study, this is unlikely to have influenced our results.

We included participants with painful mild to moderate radiographic osteoarthritis of the medial knee compartment and a neutral to varus knee alignment. The participants may have had a heterogeneous response to lateral wedge insoles depending on factors such as radiographic severity of osteoarthritis thereby masking our ability to find a significant treatment effect. Indeed although case series have suggested greater pain relief with wedge insoles in participants with milder radiographic severity, ${ }^{544}$ detection of a structural effect in this group may not have been possible in our relatively short follow-up time frame of one year. Conversely, those with moderate disease who show greater rates of structural deterioration ${ }^{45}$ may

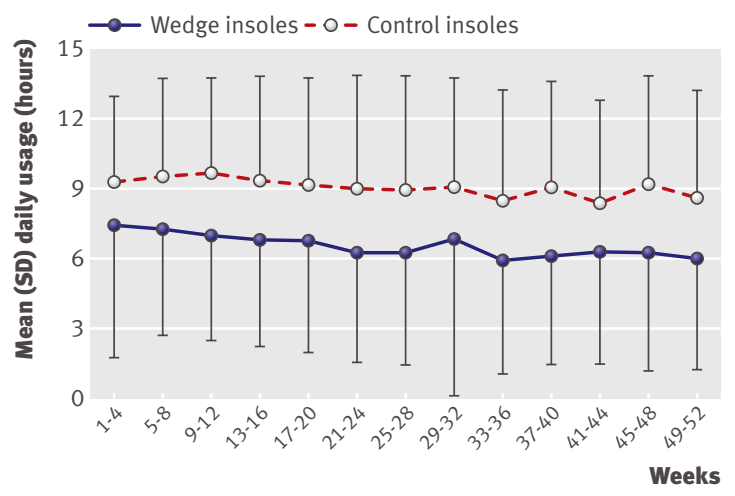

Fig 2 Mean (standard deviation) daily hours of insole use over 52 weeks 
Table $4 \mid$ Adverse events, comfort from and compliance with insole use, drug use, and cointerventions according to insole group. Values are numbers (percentages) unless stated otherwise

\begin{tabular}{|c|c|c|}
\hline Measures & $\begin{array}{l}\text { Wedge insoles } \\
\qquad(n=89)\end{array}$ & $\begin{array}{c}\text { Control insoles } \\
\qquad(n=90)\end{array}$ \\
\hline Self reported problems with insoles: & $42(47)$ & $21(23)$ \\
\hline Back pain & $9(10)$ & $1(1)$ \\
\hline Foot pain & $32(36)$ & $14(16)$ \\
\hline Uncomfortable or difficulty fitting in shoes & $15(16)$ & $4(4)$ \\
\hline Increased knee pain & $2(2)$ & $5(6)$ \\
\hline Felt unstable & $0(0)$ & $1(1)$ \\
\hline Mean (SD) insole comfort rating $(0-10)^{*}$ & $3.7(3.3)$ & $2.0(2.3)$ \\
\hline Discomfort rating: & $(n=76)$ & $(n=87)$ \\
\hline None & $40(53)$ & $64(74)$ \\
\hline Mild & $23(30)$ & $20(23)$ \\
\hline Moderate & $6(8)$ & $2(2)$ \\
\hline Severe & $5(7)$ & $1(1)$ \\
\hline Very severe & $2(3)$ & $0(0)$ \\
\hline Mean (SD) self adherence rating $(0-10) \dagger$ & $6.7(3.6)$ & $8.5(2.4)$ \\
\hline Drug use & $47(53)$ & $47(52)$ \\
\hline \multicolumn{3}{|l|}{ Drug types: } \\
\hline Analgesics & $27(30)$ & $29(32)$ \\
\hline Non-steroidal anti-inflammatory drugs & $18(20)$ & $22(24)$ \\
\hline Cyclo-oxygenase- 2 inhibitors & $5(6)$ & $1(1)$ \\
\hline Glucosamine & $7(8)$ & $7(8)$ \\
\hline Unspecified & $6(7)$ & $1(1)$ \\
\hline Cointerventions & 17 (19) & $14(16)$ \\
\hline \multicolumn{3}{|l|}{ Cointervention type: } \\
\hline Physiotherapy & $9(10)$ & $7(8)$ \\
\hline Exercise & $3(3)$ & $0(0)$ \\
\hline Cortisone injection & $2(2)$ & $1(1)$ \\
\hline Chiropractic & $2(2)$ & $2(2)$ \\
\hline Acupuncture & $2(2)$ & $0(0)$ \\
\hline Osteopathy & $2(2)$ & $0(0)$ \\
\hline Hydrotherapy & $1(1)$ & $0(0)$ \\
\hline Podiatry & $1(1)$ & $0(0)$ \\
\hline Arthroscopic surgery & $0(0)$ & $3(3)$ \\
\hline Total knee replacement & $0(0)$ & $1(1)$ \\
\hline Other & $2(2)$ & $2(2)$ \\
\hline
\end{tabular}

*Based on 11 point numerical rating scale: $0=$ extremely comfortable and $10=$ extremely uncomfortable. †Based on 11 point numerical rating scale: $0=$ not worn at all and $10=$ worn as instructed.

not respond to the use of a minor biomechanical intervention such as lateral wedge insoles. However, when we examined our subgroups with mild osteoarthritis (Kellgren and Lawrence grade 2) compared with moderate osteoarthritis (Kellgren and Lawrence grade 3) the direction of treatment effect did not differ, suggesting that the lateral wedge insoles were not having a beneficial effect in either group (data not shown).

Given that lateral wedge insoles reduce knee load, it is often assumed that this will translate to pain relief. Our finding that pain remained unchanged does not support this commonly held belief. Although some studies have shown a positive relation between severity of pain and knee load, ${ }^{4647}$ others have found no such relation ${ }^{4849}$ or even an inverse one. ${ }^{5051}$ The multiple mechanisms contributing to the experience of pain with knee osteoarthritis possibly explain our findings and those of others. For example, pain is influenced by a myriad of psychosocial factors that can vary between people as well as within people over time. ${ }^{52}$ Hence it is not surprising that favourable biomechanical changes do not guarantee pain reduction.

The optimal dosage of insole use needed for beneficial effects on pain and joint structure is not known. It has been reported that 5-10 hours of daily use produces better symptomatic outcomes than less than five hours or more than 10 hours of use. ${ }^{53}$ Although in our study the mean daily usage of 6.8 (SD 4.3) hours per day reported in the lateral wedge insole group was less than the 9.1 (SD 3.8) hours per day for the control insole group, it still fell within the range of usage recommended by a previous study. ${ }^{53}$ Furthermore, analysis using a hypothetical scenario with complete adherence to the insoles also showed no significant group differences for symptomatic or structural outcomes, suggesting that adherence was not a factor explaining our results.

Finally, we cannot exclude the possibility that our non-significant results for symptoms could reflect a type II error. Pain was only measured at baseline and at 12 months of follow-up. Knowing that pain fluctuates in patients with osteoarthritis, we may have been able to detect a significant insole effect had we included more measurement time points throughout the 12 months to better estimate the average pain over the duration of the trial.

\section{Comparison with previous studies}

Our findings are in agreement with the limited number of other randomised controlled trials that have also failed to show the efficacy of lateral wedge insoles. ${ }^{7-10}$ One study found that customised lateral heel wedge insoles worn for two years were associated with a reduced intake of non-steroidal anti-inflammatory drugs, a secondary outcome, but did not alter pain, stiffness, function, or joint space narrowing on radio graphy. ${ }^{910} \mathrm{~A}$ crossover trial found no statistical or clinical effect of a lateral wedge insole worn for six weeks. ${ }^{8}$ Similarly, in the most recent study, a customised lateral wedge insole worn in standardised walking shoes had no greater effect on pain over 12 months than a neutral insole. ${ }^{7}$ Using the largest cohort of participants to date, our results concur with these clinical trials and extend their findings by providing novel data relating to the effects of lateral wedge insoles on structural features seen on magnetic resonance imaging.

\section{Strengths and weaknesses of the study}

A strength of our randomised controlled trial was its pragmatic nature whereby the intervention was executed in a manner consistent with current clinical practice. Participants were provided with standardised off the shelf wedge insoles and permitted to wear them in their own shoes. Other strengths of our study include the rigorous study design, incorporating blinding of both assessor and participant, adequate statistical power, excellent participant retention, and recruitment of an osteoarthritis subgroup most likely to 


\section{WHAT IS ALREADY KNOWN ON THIS TOPIC}

Lateral wedge insoles are an inexpensive, easily available conservative treatment for medial knee osteoarthritis

Lateral wedge insoles reduce medial knee load, a risk factor for disease progression, but their effect on knee joint structure is unclear

13 of 14 clinical guidelines currently recommend lateral wedge insoles for symptomatic benefit of knee osteoarthritis

\section{WHAT THIS STUDY ADDS}

Lateral wedge insoles used daily for 12 months provided no additional benefit over flat control insoles in alleviating symptoms of medial knee osteoarthritis

The results do not support current clinical guidelines for knee osteoarthritis

Changes in joint structure did not differ between the groups, suggesting a lack of evidence for a disease modifying effect of lateral wedge compared with control insoles

benefit from a lateral wedge insole. Importantly, we tested a lateral wedge insole that had proved effectiveness in reducing knee load and incorporated a sensitive outcome measure (magnetic resonance imaging) to assess change in specific joint structural features. A limitation was the need to use a different magnetic resonance imaging machine for follow-up assessment in about $30 \%$ of the participants, which may have increased the error within the measurements. However, our repeatability study showed that differences in measurements between the two machines were essentially comparable to those expected with repeated measurements taken from the same machine. Furthermore, we also recalculated all of the primary and secondary endpoint analyses assessing whether the estimated intervention effect differed for participants who were scanned with one machine compared with those scanned with both machines, but we found no evidence of such differences (all interaction $P$ values $>0.15$ ). Our participants were volunteers recruited from the community, which is a common method of recruitment for trials of non-drug conservative interventions in knee osteoarthritis. It is possible that our sample does not reflect fully the patients who seek treatment from general medical practitioners. These patients are likely to exhibit more severe disease and thus could respond differently to treatment with lateral wedge insoles. They may also differ in terms of psychosocial factors that are known to influence symptoms and could affect outcomes from treatment. Our results can only be generalised to other lateral wedge insoles with similar design features. Given that some evidence exists for subtalar strapping enhancing the effectiveness of lateral wedge insoles, ${ }^{5455}$ this type of insole warrants further investigation.

\section{Conclusions and practice implications}

In summary, our findings showed that lateral wedge insoles had no effect on symptoms or disease progression over 12 months in people with mild to moderate medial knee osteoarthritis. These findings have implications for clinical practice. Given that most clinical guidelines currently recommend lateral wedge insoles for improving pain and function, our results and those of other randomised controlled trials suggest that such insoles are not beneficial for the treatment of symptoms. However, given the clear biomechanical benefits of wedge insoles in reducing medial knee load, further research is needed over a longer time frame to conclusively determine the effects of lateral wedge insoles on joint structure.

We thank the project staff, including Ben Metcalf and Georgina Morrow, who assisted with recruitment and database management and Joanna Barrett who assisted with the podiatry screening.

Contributors: KLB and RSH conceived and designed the trial protocol. $\mathrm{KLB}, \mathrm{RSH}, \mathrm{FC}, \mathrm{AH}$, and $\mathrm{CP}$ procured the project funding. CP was responsible for the podiatric screening assessment and insole design, acquisition, and fitting. KLB and RSH contributed to clinical screening of participants and graded the $x$ ray films. MD-T and FH analysed the magnetic resonance imaging scans overseen by FC. AF and EW did the statistical analyses. K-AB recruited the participants, measured knee alignment, and carried out the outcome assessments. KLB and RSH drafted the manuscript, and $\mathrm{K}-\mathrm{AB}, \mathrm{AF}, \mathrm{EW}, \mathrm{CP}, \mathrm{FC}, \mathrm{FH}, \mathrm{AH}$, and MD-T contributed to the manuscript. All authors read and approved the final manuscript. KLB and RSH accept full responsibility for this work and act as guarantors for the study.

Funding: This study was supported by a project grant from the National Health and Medical Research Council (No 350297). KLB is funded in part by an Australian Research Council future fellowship. The study sponsor had no role in the design of the study; the collection, analysis, and interpretation of the data; or the writing of the article and the decision to submit it for publication.

Competing interests: All authors have completed the Unified Competing Interest form at www.icmje.org/coi_disclosure.pdf (available on reques from the corresponding author) and declare: KLB is partly supported by a fellowship from the Australian Research Council; KLB and RSH have an Australian Research Council linkage grant with an industry partner ASICS Oceania to develop and test a modified shoe to reduce medial knee load no other relationships or activities that could appear to have influenced the submitted work.

Ethical approval: This study was approved by the University of Melbourne Human Research Ethics Committee and by the Department of Human Services Victoria, Radiation Safety Committee.

Data sharing: The statistical code and dataset are available from the corresponding author at k.bennell@unimelb.edu.au.

1 Lawrence RC, Felson DT, Helmick CG, Arnold LM, Choi H, Deyo RA, et al. Estimates of the prevalence of arthritis and other rheumatic conditions in the United States. Part II. Arth Rheum 2008;58:26-35.

2 Zhang W, Moskowitz RW, Nuki G, Abramson S, Altman RD, Arden N, et al. OARSI recommendations for the management of hip and knee osteoarthritis, part II: OARSI evidence-based, expert consensus guidelines. Osteo \& Cart 2008;16:137.

3 Lachance L, Sowers MF, Jamadar D, Hochberg M. The natural history of emergent osteoarthritis of the knee in women. Osteo \& Cart 2002;10:849-54.

4 Miyazaki T, Wada M, Kawahara H, Sato M, Baba H, Shimada S. Dynamic load at baseline can predict radiographic disease progression in medial compartment knee osteoarthritis. Ann Rheum Dis 2002;61:617-22.

5 Hinman RS, Payne C, Metcalf BR, Wrigley TV, Bennell KL. Lateral wedges in knee osteoarthritis: what are their immediate clinical and biomechanical effects and can these predict a three-month clinical outcome? Arth Rheum 2008;59:408-15.

6 Kerrigan DC, Lelas JL, Goggins J, Merriman GJ, Kaplan RJ, Felson DT. Effectiveness of a lateral-wedge insole on knee varus torque in patients with knee osteoarthritis. Arch Phys Med Rehabil 2002;83:889-93.

7 Barrios JA, Crenshaw JR, Royer TD, Davis IS. Walking shoes and laterally wedged orthoses in the clinical management of medial tibiofemoral osteoarthritis: a one-year prospective controlled trial. Knee 2009;16:136-42.

8 Baker K, Goggins J, Xie H, Szumowski K, LaValley M, Hunter DJ, et al. A randomized crossover trial of a wedged insole for treatment of knee osteoarthritis. Arth Rheum 2007;56:1198-203.

9 Pham T, Maillefert JF, Hudry C, Kieffert P, Bourgeois P, Lechevalier D, et al. Laterally elevated wedged insoles in the treatment of medial knee osteoarthritis. A two-year prospective randomized controlled study. Osteo \& Cart 2004;12:46-55. 
10 Maillefert JF, Hudry C, Baron G, Kieffert P, Bourgeois P, Lechevalier D, et al. Laterally elevated wedged insoles in the treatment of medial knee osteoarthritis: a prospective randomized controlled study. Osteo \& Cart 2001;9:738-45.

11 Reichmann WM, Maillefert JF, Hunter DJ, Katz JN, Conaghan PG, Losina E. Responsiveness to change and reliability of measurement of radiographic joint space width in osteoarthritis of the knee: a systematic review. Osteo \& Cart 2011: published online 23 March.

12 Hunter DJ, Zhang W, Conaghan PG, Hirko K, Menashe L, Reichmann WM, et al. Responsiveness and reliability of MRI in knee osteoarthritis: a meta-analysis of published evidence. Osteo \& Cart 2011: published online 9 March.

13 Hinman RS, Bowles KA, Payne C, Bennell KL. Effect of length on laterally-wedged insoles in knee osteoarthritis. Arth Rheum 2008;59:144-7.

14 Altman RD, Hochberg M, Murphy WA Jr, Wolfe F, Lequesne M. Atlas of individual radiographic features in osteoarthritis. Osteo \& Cart 1995;3(suppl A):3-70.

15 Kraus VB, Vail TP, Worrell T, McDaniel G. A comparative assessment of alignment angle of the knee by radiographic and physical examination methods. Arth Rheum 2005;52:1730-5.

16 Kellgren JH, Lawrence JS. Radiological assessment of osteo-arthrosis. Ann Rheum Dis 1957;16:494-502.

17 Hunter DJ, Harvey W, Gross KD, Felson D, McCree P, Li L, et al. A randomized trial of patellofemoral bracing for treatment of patellofemoral osteoarthritis. Osteo \& Cart 2011: published online 11 January.

18 Bennell K, Bowles KA, Payne C, Cicuttini F, Osborne R, Harris A, et al. Effects of laterally wedged insoles on symptoms and disease progression in medial knee osteoarthritis: a protocol for a randomised, double-blind, placebo controlled trial. BMC Musc Dis 2007;8:96.

19 Bellamy N. Osteoarthritis clinical trials: candidate variables and clinimetric properties. J Rheum 1997;24:768-78.

20 Wandel S, Juni P, Tendal B, Nuesch E, Villiger PM, Welton NJ, et al. Effects of glucosamine, chondroitin, or placebo in patients with osteoarthritis of hip or knee: network meta-analysis. $B M$ 2010;341:c4675.

21 Salaffi F, Stancati A, Silvestri CA, Ciapetti A, Grassi W. Minimal clinically important changes in chronic musculoskeletal pain intensity measured on a numerical rating scale. Eur J Pain 2004;8:283-91.

22 Bellamy N, Kirwan J, Boers M, Brooks P, Strand V, Tugwell P, et al. Recommendations for a core set of outcome measures for future phase III clinical trials in knee, hip, and hand osteoarthritis. Consensus development at OMERACT III. J Rheum 1997;24:799-802.

23 Bellamy N, Buchanan WW, Goldsmith CH, Campbell J, Stitt LW. Validation study of WOMAC: a health status instrument for measuring clinically important patient relevant outcomes to antirheumatic drug therapy in patients with osteoarthritis of the hip or knee. J Rheum 1988;15:1833-40.

24 Hawthorne G, Richardson J, Osborne R. The Assessment of Quality of Life (AQoL) instrument: a psychometric measure of health-related quality of life. Qual Life Res 1999;8:209-24.

25 Washburn RA, Ficker JL. Physical Activity Scale for the Elderly (PASE) the relationship with activity measured by a portable accelerometer. J Sports Med Phys Fitness 1999;39:336-40.

26 Wluka AE, Davis SR, Bailey M, Stuckey SL, Cicuttini FM. Users of oestrogen replacement therapy have more knee cartilage than nonusers. Ann Rheum Dis 2001;60:332-6.

27 Wluka AE, Stuckey S, Snaddon J, Cicuttini FM. The determinants of change in tibial cartilage volume in osteoarthritic knees. Arth Rheum 2002;46:2065-72

28 Cicuttini FM, Wluka AE, Forbes A, Wolfe R. Comparison of tibial cartilage volume and radiologic grade of the tibiofemoral joint. Arth Rheum 2003;48:682-8.

29 Cicuttini F, Ding C, Wluka A, Davis S, Ebeling PR, Jones G. Association of cartilage defects with loss of knee cartilage in healthy, middle-age adults: a prospective study. Arth Rheum 2005;52:2033-9.

30 Ding C, Garnero P, Cicuttini F, Scott F, Cooley H, Jones G. Knee cartilage defects: association with early radiographic osteoarthritis, decreased cartilage volume, increased joint surface area and type II collagen breakdown. Osteo \& Cart 2005;13:198-205.

31 Davies-Tuck ML, Wluka AE, Wang Y, Teichtahl AJ, Jones G, Ding C, et al. The natural history of cartilage defects in people with knee osteoarthritis. Osteo \& Cart 2008;16:337-42.

32 Wluka AE, Hanna F, Davies-Tuck M, Wang Y, Bell RJ, Davis SR, et al. Bone marrow lesions predict increase in knee cartilage defects and loss of cartilage volume in middle-aged women without knee pain over 2 years. Ann Rheum Dis 2009;68:850-5.
33 Tanamas SK, Wluka AE, Pelletier JP, Pelletier JM, Abram F, Berry PA, et al. Bone marrow lesions in people with knee osteoarthritis predict progression of disease and joint replacement: a longitudinal study. Rheumatology (Oxford) 2010;49:2413-9.

34 Bellamy N, Carette S, Ford PM, Kean WF, le Riche NG, Lussier A, et al. Osteoarthritis antirheumatic drug trials. III. Setting the delta for clinical trials-results of a consensus development (Delphi) exercise. Rheum 1992;19:451-7.

35 Bennell KL, Hinman RS, Metcalf BR, Buchbinder R, McConnell J, McColl G, et al. Efficacy of physiotherapy management of knee joint osteoarthritis: a randomised, double blind, placebo controlled trial. Ann Rheum Dis 2005;64:906-12.

36 Hinman RS, Crossley KM, McConnell J, Bennell KL. Efficacy of knee tape in the management of osteoarthritis of the knee: blinded randomised controlled trial. BMJ 2003;327:135.

37 Cicuttini FM, Jones G, Forbes A, Wluka AE. Rate of cartilage loss at two years predicts subsequent total knee arthroplasty: a prospective study. Ann Rheum Dis 2004;63:1124-7.

38 White IR, Thompson SG. Adjusting for partially missing baseline measurements in randomized trials. Stat Med 2005;24:993-1007.

39 Little RJA, Rubin DB. Statistical analysis with missing data. John Wiley, 1987.

40 Stuart EA, Perry DF, Le HN, lalongo NS. Estimating intervention effects of prevention programs: accounting for noncompliance. Prev Sci 2008;9:288-98.

41 Cicuttini F, Wluka A, Hankin J, Wang Y. Longitudinal study of the relationship between knee angle and tibiofemoral cartilage volume in subjects with knee osteoarthritis. Rheumatology (Oxford) 2004;43:321-4.

42 Eckstein F, Cicuttini F, Raynauld JP, Waterton JC, Peterfy C. Magnetic resonance imaging (MRI) of articular cartilage in knee osteoarthritis (OA): morphological assessment. Osteo \& Cart 2006;14(suppl A):A46-75.

43 Toda Y, Tsukimura N. Influence of concomitant heeled footwear when wearing a lateral wedged insole for medial compartment osteoarthritis of the knee. Osteo \& Cart 2008;16:244-53.

44 Keating EM, Faris PM, Ritter MA, Kane J. Use of lateral heel and sole wedges in the treatment of medial osteoarthritis of the knee. Orthop Rev 1993;22:921-4.

45 Eckstein F, Nevitt M, Gimona A, Picha K, Lee JH, Davies RY, et al. Rates of change and sensitivity to change in cartilage morphology in healthy knees and in knees with mild, moderate, and end stage radiographic osteoarthritis. Arthritis Care Res (Hoboken) 2010: published online 18 October.

46 Kito N, Shinkoda K, Yamasaki T, Kanemura N, Anan M, Okanishi N, et al. Contribution of knee adduction moment impulse to pain and disability in Japanese women with medial knee osteoarthritis. Clin Biomech (Bristol, Avon) 2010;25:914-9.

47 Thorp LE, Sumner DR, Wimmer MA, Block JA. Relationship between pain and medial knee joint loading in mild radiographic knee osteoarthritis. Arth Rheum 2007;57:1254-60.

48 Maly MR, Costigan PA, Olney SJ. Mechanical factors relate to pain in knee osteoarthritis. Clin Biomech (Bristol, Avon) 2008;23:796-805.

49 Hurwitz DE, Ryals AB, Case JP, Block JA, Andriacchi TP. The knee adduction moment during gait in subjects with knee osteoarthritis is more closely correlated with static alignment than radiographic disease severity, toe out angle and pain. J Orthop Res 2002;20:101-7.

50 Briem K, Axe MJ, Snyder-Mackler L. Medial knee joint loading increases in those who respond to hyaluronan injection for medial knee osteoarthritis. J Orthop Res 2009;27:1420-5.

51 Hurwitz DE, Ryals AR, Block JA, Sharma L, Schnitzer T], Andriacchi TP. Knee pain and joint loading in subjects with osteoarthritis of the knee. J Orthop Res 2000;18:572-9.

52 Somers TJ, Keefe FJ, Godiwala N, Hoyler GH. Psychosocial factors and the pain experience of osteoarthritis patients: new findings and new directions. Curr Opin Rheumatol 2009;21:501-6.

53 Toda Y, Tsukimura N, Segal N. An optimal duration of daily wear for an insole with subtalar strapping in patients with varus deformity osteoarthritis of the knee. Osteo \& Cart 2005;13:353-60.

54 Toda Y, Tsukimura N. A 2-year follow-up of a study to compare the efficacy of lateral wedged insoles with subtalar strapping and inshoe lateral wedged insoles in patients with varus deformity osteoarthritis of the knee. Osteo \& Cart 2006;14:231-7.

55 Toda Y, Tsukimura N. A six-month followup of a randomized trial comparing the efficacy of a lateral-wedge insole with subtalar strapping and an in-shoe lateral-wedge insole in patients with varus deformity osteoarthritis of the knee. Arth Rheum 2004;50:3129-36.

Accepted: 30 March 201 